\title{
Characterization of a highly efficient chevron-shaped anti-contamination device
}

\author{
M. Fiore ${ }^{1,2} \cdot$ O. Vermeersch ${ }^{1,2} \cdot$ M. Forte ${ }^{1,2} \cdot$ G. Casalis $^{1,2} \cdot$ C. François $^{1,3}$
}

\begin{abstract}
This paper is devoted to the characterization of an optimized chevron-shaped anti-contamination device (ACD). This device can prevent efficiently the propagation of turbulence from the fuselage along the attachment line (hypothetical streamline that spreads the flow going to suction side and the one going to pressure side) of swept wings and enables the development of a new laminar boundary layer downstream. More specifically, the aim is to prevent boundary-layer transition along the attachment line by a contamination process. This process is characterized by the typical Reynolds number $\bar{R}$ and the associated Poll's criterion. Thus, ACD efficiency will be expressed in terms of $\bar{R}$ values. Some experiments performed on a new numerically optimized ACD have shown its ability to prevent leading-edge contamination up to $\bar{R}$ values close to the natural transition process of the laminar boundary layer along the attachment line. The corresponding stability analysis of the laminar boundary layer is made using the Görtler-Hämmerlin stability approach. The study is completed with the different transition processes that can occur downstream the attachment line, around the airfoil, especially with crossflow analysis.
\end{abstract}

O. Vermeersch

olivier.vermeersch@onera.fr

1 ONERA - The French Aerospace Lab, Toulouse 31055, France

2 Aerodynamics and Energetics Modelling Department, ONERA, 2 Avenue Edouard Belin, Toulouse 31055, France

3 Applied Aerodynamics Department, ONERA, 8 rue des Vertugadins, Meudon 92190, France

\section{Introduction}

Reducing the extent of turbulent boundary layer on aircraft is of considerable interest because it lowers friction drag, fuel consumption and its related pollutant emission. More precisely, progress has been made to maintain as far as possible a laminar flow around tail planes and wings of commercial aircraft. These efforts can be annihilated by leading-edge contamination which can occur on swept wings. Turbulence convected along the fuselage propagates along the attachment line and spreads over the wing surface leading to the transition of the boundary layer. Theoretical developments (see Görtler 1955; Pfenninger 1965; Poll 1978) have led to the definition of a quantity called «Reynolds bar» and denoted $\bar{R}$ to establish if contamination process is likely to occur depending on the value of this quantity (threshold). In standard flight conditions, $\bar{R}$ for commercial aircraft is often higher than the critical threshold and the leading-edge contamination may happen. In order to address this issue, Gaster (1965) designed a passive device able to stop turbulence coming from wind tunnel wall that simulated the fuselage. This small fairing, located close to the wing root, was made in such a way that contaminating turbulent boundary layer was brought to face with a stagnation point, while a «clean» laminar boundary layer developed downstream. This «Gaster bump» or anticontamination device (ACD) was able to prevent leadingedge contamination up to $\bar{R}$ values higher than the critical threshold in smooth condition $\bar{R}=250$, but the gain remained modest.

In 1967, after some wind tunnel experiments, Gaster reported a maintenance of a laminar flow downstream this bump along the attachment line up to $\bar{R}=420$. More recent in-flight experiments on Falcon 50 and 900 (see Arnal 1996) have shown similar results for a standard Gaster 
bump set-up on wings allowing to maintain laminarity up to $\bar{R}$ values between 350 and 400 . These values showed an improvement relatively to the contamination criterion without ACD $(\bar{R}=250)$. However, values of $\bar{R}$ on lifting surfaces of civil aircraft range from 400 to 700 near the wing root. Therefore, a clear improvement in ACD efficiency had to be achieved for civil aircraft applications.

Krier and Sucipto (2009) and Krier et al. (2009) were the first to propose an arrow-shaped ACD in the framework of the SUPERTRAC European project (SUPersonic TRANsition Control). The ACD design by Krier et al was made of a cylindrical nose prolonged by streamlined legs in order to alter as less as possible the flow in the attachment line region. This shape was successfully tested in a supersonic wind tunnel for two Mach numbers $M=1.7$ and $M=2.7$. This ACD was able to delay contamination up to $\bar{R}=330$ at $M=1.7$ and $\bar{R}=400$ at $M=2.7$. After these experiments, Euler computations have shown the possibility of such devices to delay contamination for lower speeds and thus their potential application in transonic commercial aircraft.

Since several decades, ONERA has acquired a strong expertise in the design and testing of ACDs (see Arnal et al. 1996; Gasparian 1998) that has led to the development of a very efficient ACD experimentally tested in 2014 and detailed through this article. These research activities have started in 2010 in the framework of the Joint Technology Initiative Clean Sky Project and have enabled the development of an optimized chevron-shaped ACD specifically designed for low-speed conditions.

Section 2 will introduce general knowledge about flow characteristics related to a swept wing with wall junction. This basis will enable further developments on leading-edge contamination characterization. When this phenomenon can be avoided, for example, by using an ACD, the laminar boundary layer that develops downstream can naturally become unstable. This second attachment line transition process will also be addressed. The third section will be dedicated to ACD specifications and development performed by ONERA in order to optimize its ability to prevent leadingedge contamination. Then, the experimental set-up will be introduced in Sect. 4. Finally, results related to this test campaign will be detailed, especially concerning destabilizing process characteristics and ACD performance validation.

\section{Mean flow and transition processes around swept wings}

\subsection{Mean flow}

This section gives a general overview about swept-wing flow characteristics. Mean flow along the attachment line of a swept wing (sweep angle $\varphi$ ) is the three-dimensional

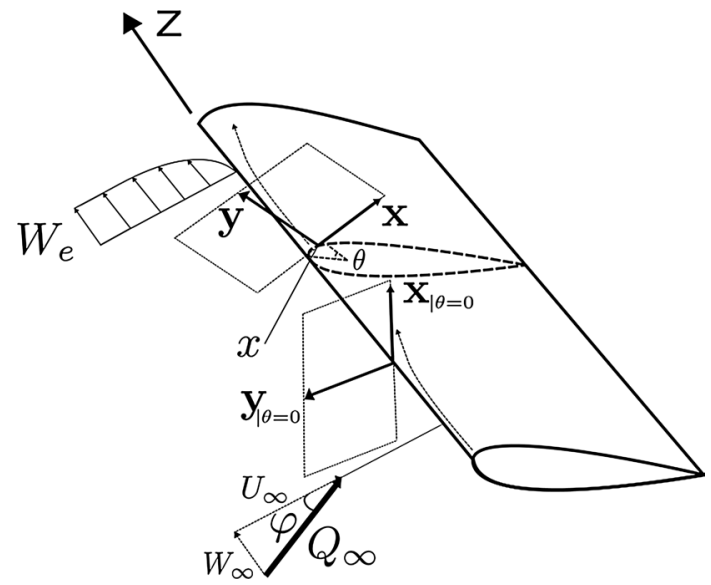

Fig. 1 Swept-wing flow characteristics

swept stagnation flow. It consists of the superposition of the Hiemenz (1911) plane stagnation flow $[(\mathbf{x}, \mathbf{y})$ with $\mathbf{x}$ chordwise direction and $\mathbf{y}$ wall normal direction] and a transverse uniform velocity in the span direction $\mathbf{Z}$ (see Fig. 1). The coordinate $x$ will be used as the curvilinear abscissa around the airfoil with the origin $x=0$ on the attachment line. The coordinate $y$ is the distance from the wall in its normal direction. Under the assumption of an infinite swept wing $(\partial / \partial Z=0)$, velocity components in the boundary layer $(U, V, W)$ in the $(\mathbf{x}, \mathbf{y}, \mathbf{Z})$ spatial directions are formulated using self-similarity functions $(f, g)$ defined as:

$U_{e}(x, y)=k x \quad V_{e}(x, y)=-k y \quad W_{e}(x, y)=W_{\infty}$

$\frac{U}{U_{e}}=f^{\prime}(\eta) \quad \frac{V}{V_{e}}=-f(\eta) \quad \frac{W}{W_{e}}=g^{\prime}(\eta)$

with $\eta=\frac{y}{\Delta} \quad$ and $\quad \Delta=\sqrt{\frac{\nu}{k}}$

where $\left(U_{e}, V_{e}, W_{e}\right)$ are the velocity components at the edge of the boundary layer in the $(\mathbf{x}, \mathbf{y}, \mathbf{Z})$ directions. $W_{\infty}=$ $Q_{\infty} \sin (\varphi)$ is the far-field velocity component in the span direction Z. $k$ and $v$ represent respectively a strain rate and the kinematic viscosity of the fluid. Self-similarity functions are resolved from the following ordinary differential equations obtained for incompressible flows:

$f^{\prime \prime}+f^{\prime \prime} f-f^{\prime 2}+1=0$

$g^{\prime \prime}+g^{\prime} f=0$

with boundary conditions imposed at the wall $(\eta=0)$ and in far-field direction $(\eta \rightarrow+\infty)$ :

$f(0)=0 \quad f^{\prime}(0)=0 \quad g(0)=0 \quad g^{\prime}(0)=0$

$\lim _{\eta \rightarrow+\infty} f^{\prime}(\eta)=1 \quad \lim _{\eta \rightarrow+\infty} g^{\prime}(\eta)=1$ 


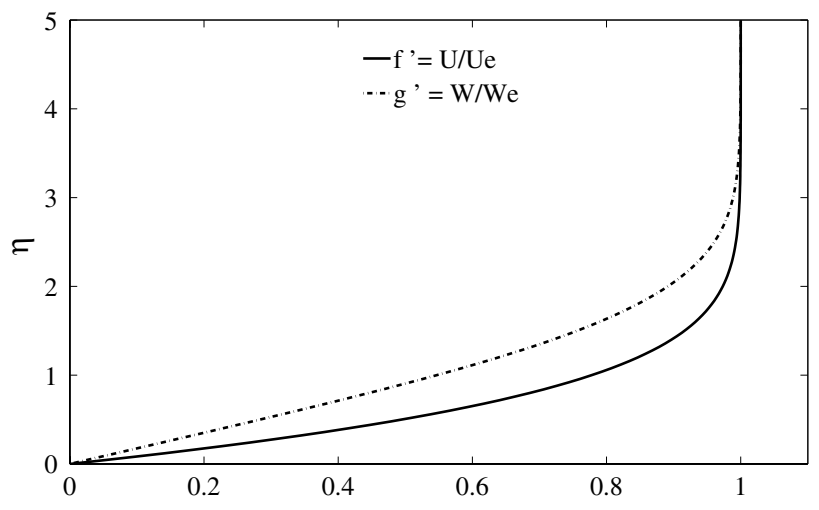

Fig. 2 Falkner-Skan-Cooke solutions

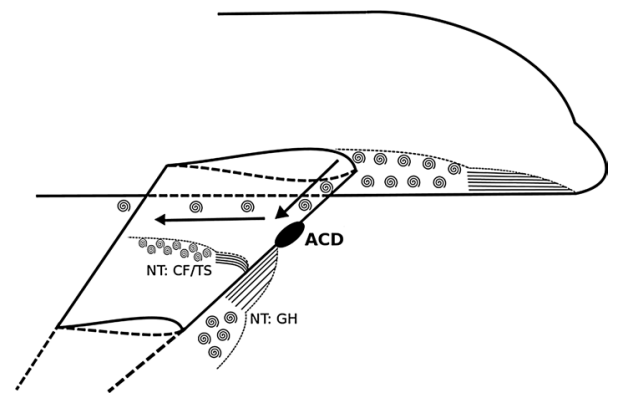

Fig. 3 Possible path to turbulence on a swept wing for a non-contaminated configuration (with efficient $\mathrm{ACD}$ ). $N T$ natural transition

Based on the latter Falkner-Skan-Cooke self-similarity functions (see Fig. 2), Reynolds number from displacement, momentum thicknesses $\left(R_{\delta_{1}}, R_{\theta}\right)$ and shape factor $(H)$ can then be deduced:

$R_{\delta_{1}}=\frac{W_{e} \delta_{1}}{v} \quad$ with $\quad \delta_{1}=\Delta \int_{0}^{\delta}\left(1-g^{\prime}(\eta)\right) \mathrm{d} \eta$

$R_{\theta}=\frac{W_{e} \theta}{v} \quad$ with $\quad \theta=\Delta \int_{0}^{\delta} g^{\prime}(\eta)\left(1-g^{\prime}(\eta)\right) \mathrm{d} \eta$

$H=\frac{\delta_{1}}{\theta}$

We will see in the next paragraph that latter quantities can be formulated more simply using $\bar{R}$.

In laminar-turbulent transition process along the attachment line of a swept wing, mainly two transition processes may occur: either a leading-edge contamination (see Sect. 2.2) that can be linked to a bypass transition, or when contamination can be prevented (by an ACD for example), the new laminar boundary layer that develops downstream can also become turbulent due to a natural transition process involving Görtler-Hämmerlin
(GH) instabilities (see Fig. 3). In the first case, transition along the attachment line is driven by non linear interactions with the fuselage turbulent boundary layer, while the second process can be described using a linear stability approach (see Sect. 2.3).

\subsection{Leading-edge contamination}

Many experiments concerning leading-edge contamination phenomenon have been carried out and have highlighted a limited number of relevant parameters. These parameters are gathered in the quantity $\bar{R}$ that can be formulated as:

$\bar{R}=\frac{W_{e} \Delta}{v}$

At low speed, Pfenninger (1965) and Poll (1978) have developed a simple criterion (referred as Poll's criterion) based on the value of $\bar{R}$ to establish if leading-edge contamination is likely to occur:

If $\bar{R} \leq 250$, the bursts of turbulence convected along the attachment line are damped and vanish as they travel along the attachment line.

For $\bar{R} \geq 250$, bursts are self-sustaining. They grow and overlap so that the attachment line boundary layer becomes fully turbulent.

Poll (1985) extended this criterion to high-speed flows by introducing a modified length scale $\eta^{*}$ and a modified Reynolds number $\overline{R^{*}}$ which have the same definition than $\eta$ and $\bar{R}$, except that $v$ is replaced by $v^{*}$. The latter quantity is the kinematic viscosity computed at a reference temperature $T^{*}$ which can be estimated from an empirical relationship:

$T^{*}=T_{\mathrm{e}}+A\left(T_{\mathrm{w}}-T_{\mathrm{e}}\right)+B\left(T_{\mathrm{aw}}-T_{\mathrm{e}}\right)$

where $T_{\mathrm{e}}$ is the temperature at the boundary-layer edge, $T_{\mathrm{w}}$ is the wall temperature, and $T_{\mathrm{aw}}$ is the adiabatic wall (recovery) temperature. $A=0.1$ and $B=0.6$ are empirical constants commonly used. Correlation of existing data suggested that contamination is likely to occur for $\overline{R^{*}}=245 \pm 35$.

Reynolds numbers for displacement and momentum thicknesses can then be expressed using $\bar{R}$ by:

$R_{\delta_{1}}=1.026 \bar{R}$

$R_{\theta}=0.404 \bar{R}$

$H=2.54$

Poll's criterion threshold can then be defined in terms of momentum thickness by $R_{\theta \text {,crit }}=101$. 


\subsection{Natural transition}

When contamination phenomenon can be avoided (see Fig. 3), different processes all related to natural boundarylayer instabilities can lead to transition in different locations of the wing surface. Even if each of these different processes exhibit particularities that will be depicted in this article, some common main steps can be identified. The first step is the receptivity phase. Free-stream turbulence and noise, vibrations or small roughness elements can «enter» the laminar boundary layer and begin to excite its eigenmodes. ${ }^{1}$ Then, during the linear amplification phase, eigenmodes that take the form of periodic waves can grow or decay following a linear mechanism. Finally, some amplified modes evolve nonlinearly, so that turbulent spots appear and lead to transition. The first natural transition process that may occur when contamination can be avoided is a boundary-layer destabilization along the attachment line. As we will see, this destabilization referred as GörtlerHämmerlin instability will be studied using the threedimensional swept stagnation flow introduced previously. This approach will be of central interest for ACD efficiency characterization.

When the boundary layer can be maintained laminar along the attachment line, it can become unstable around the airfoil in chordwise direction. Depending on the location, pressure gradient can have a destabilizing effect on the boundary layer. Near the leading edge where the flow is strongly accelerated (negative pressure gradient), the crossflow mean-velocity profile $(W)$ inside the boundary layer is highly unstable. It generates crossflow instabilities with a wave-number vector making a nearly orthogonal angle with respect to the free-stream direction. This leads to the formation of co-rotating vortices, the so-called crossflow instabilities. In particular, steady modes are strongly amplified and are suspected to be the dominant modes in a low free-stream turbulent environment (e.g. in flight). Downstream, in region of decelerated flow (positive pressure gradient), the streamwise mean-velocity profile $(U)$ is unstable. Some waves, similar to two-dimensional Tollmien-Schlichting waves, are amplified with a wave-number vector nearly aligned with the free-stream direction and trigger the transition. The possibility of designing laminar wings is thus given by the ability to control these different destabilizing phenomena.

A common theoretical framework can be adopted to study the growth and destabilization of these boundarylayer modes. In the linear stability theory that will be

\footnotetext{
${ }^{1}$ Here, it is assumed that the external disturbance level is raher low so that the transition is not driven by the so-called bypass mechanism, see Morkovin et al. (1994).
}

applied, only the linear amplification step (second phase) is considered to estimate the transition location. Transition will then be given for a certain amplification value $A / A_{0}$ of a wave. A (respectively $A_{0}$ ) is the amplitude of the considered mode at a given abscissa (respectively at a reference abscissa). Wave amplification is obtained from the classical Orr-Sommerfeld theory (1908). For incompressible flow, velocity field and pressure $(\wedge)$ are decomposed into a non-dimensional base flow (-) and disturbances $\left({ }^{\prime}\right)$ :

$(\hat{U}, \hat{V}, \hat{W}, \hat{P})^{t}=(\bar{U}, \bar{V}, \bar{W}, \bar{P})^{t}+\left(u^{\prime}, v^{\prime}, w^{\prime}, \pi\right)^{t}$

$\left(\begin{array}{c}u^{\prime} \\ v^{\prime} \\ w^{\prime} \\ \pi\end{array}\right)=\left(\begin{array}{c}u(y) \\ v(y) \\ w(y) \\ \pi(y)\end{array}\right) \exp [i(\alpha x+\beta Z-\omega t)]$

where $u, v, w$, are the wave amplitudes in the $\mathbf{x}, \mathbf{y}$ and $\mathbf{Z}$ directions and $\pi$ the pressure fluctuation amplitude. $\alpha=\alpha_{r}+i \alpha_{i} \in \mathbb{C}, \beta$ and $\omega \in \mathbb{R}$ are, respectively, the wave numbers along $\mathbf{x}, \mathbf{Z}$ and the angular frequency. This formalism is adapted to the destabilization of the flow around the airfoil in the $\mathbf{x}$ direction (Tollmien-Schlichting and crossflow waves). Considering this approach as starting point, Görtler and Hämmerlin (1955) developed a stability theory for attachment line application using a particular mathematical form for the base flow and disturbance quantities:

$\bar{U}=\frac{x f^{\prime}}{\bar{R}} \quad \bar{V}=\frac{-f}{\bar{R}} \quad \bar{W}=g^{\prime}$

$\left(\begin{array}{c}u^{\prime} \\ v^{\prime} \\ w^{\prime} \\ \pi\end{array}\right)=\left(\begin{array}{c}x u(y) \\ v(y) \\ w(y) \\ \pi(y)\end{array}\right) \exp [i(\beta Z-\omega t)]$

where $\beta=\beta_{r}+i \beta_{i} \in \mathbb{C}$. The main difference with the Orr-Sommerfeld approach is the $x$ dependence of $u^{\prime}$ similarly to its correlated external velocity $U_{\mathrm{e}}$. For a considered wave (defined as its real angular frequency $\omega$ ) and a fixed $\bar{R}$, the stability theory gives access to the corresponding amplified Görtler-Hämmerlin mode. When $\beta_{i}$ is negative, according to the formulation (9b), this wave is amplified in spanwise direction and can lead to the natural transition (NT on Fig. 3).

From this attachment line stability analysis, the neutral curve (iso $\beta_{i}=0$ ) that separates stable from unstable domains can be drawn in a $(\bar{R}, \omega)$ diagram (see Fig. 4). When leading-edge contamination can be prevented, it can be noticed that the attachment line laminar boundary layer becomes unstable for $\bar{R} \geq 582$. 


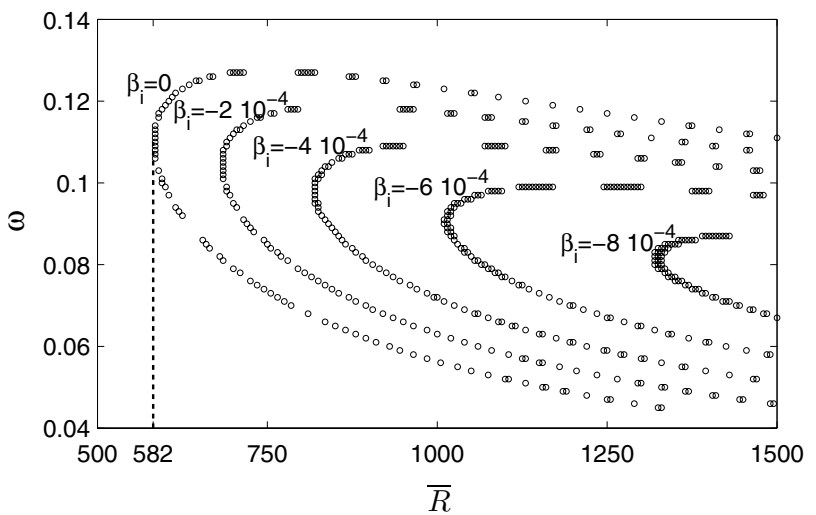

Fig. 4 iso- $\beta_{i}$ curves from the Görtler-Hämmerlin stability analysis

\section{ACD design}

Some prerequisites have to be fulfilled to guarantee the ACD efficiency (see Arnal et al. 1996). The anti-contamination process is based on a stagnation point generated by the ACD that stops the turbulent boundary layer from the fuselage and enables the downstream development of a new laminar boundary layer. To be fully efficient, the ACD has to be high enough so that the stagnation point would be located above the incoming fuselage turbulent boundary layer. Besides, the pressure distribution downstream the ACD has to allow a region of attached laminar flow. It was noticed that an increase in the radius at the top corner of the ACD and a humped shape could improve its efficiency. For aeronautic applications, the ACD has to tolerate some variations in angle of attack and sideslip angle that may occur during cruise. Finally, the ACD must have a very little impact on total drag in order to keep the potential benefits of a laminar boundary layer.

In 2010, a numerical model of the ACD mounted on a swept wing has been achieved using CATIA software. This approach enabled a parametric study of the ACD where some geometric parameters were varied: dimensions, angles and shape while keeping its ability to be put on the same swept-wing leading edge. Structured meshes were then generated using an ANSYS software. Three-dimensional Reynolds-Averaged Navier-Stokes (RANS) computations were carried out using the ONERA elsA code with future corresponding experimental conditions $P_{i 0}=101,325 \mathrm{~Pa}$, $T_{i 0}=288 \mathrm{~K}$ and $Q_{\infty}=20 \mathrm{~m} \mathrm{~s}^{-1}(\bar{R}=375)$. Efforts were made to enable the development of a boundary layer without separation on and around the ACD, especially by studying the wall streamlines (see Fig. 5). After a selection of the most promising shapes, it was decided to perform new numerical computations using laminar-turbulent transition criteria (AHD-C1, Arnal and Habiballah 1984) with an extended velocity range up to $Q_{\infty}=50 \mathrm{~m} \mathrm{~s}^{-1}(\bar{R}=590)$. This led to a new optimized shape that was tested in 2014. A sketch of

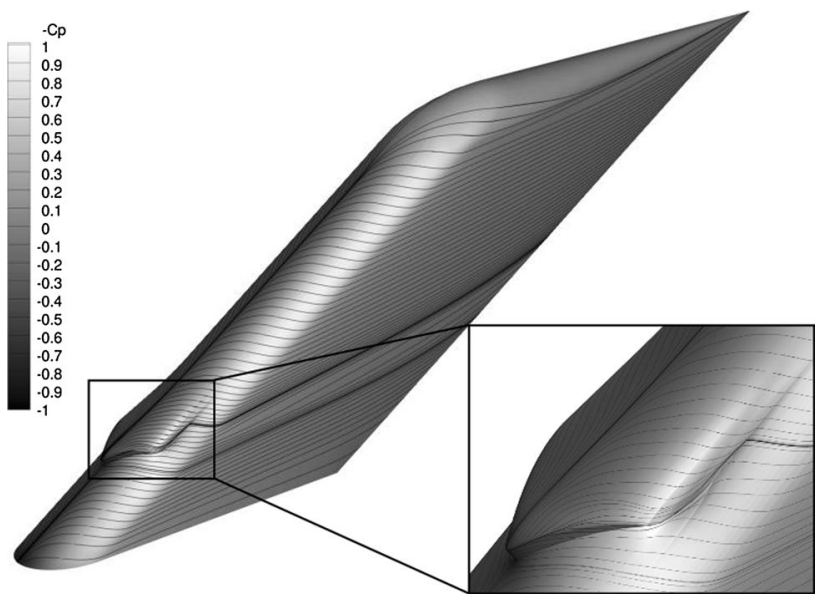

Fig. 5 Wall pressure distribution and streamlines on the swept model equipped with $\mathrm{ACD}$ at $Q_{\infty}=20 \mathrm{~m} \mathrm{~s}^{-1}$ (numerical post-processing)

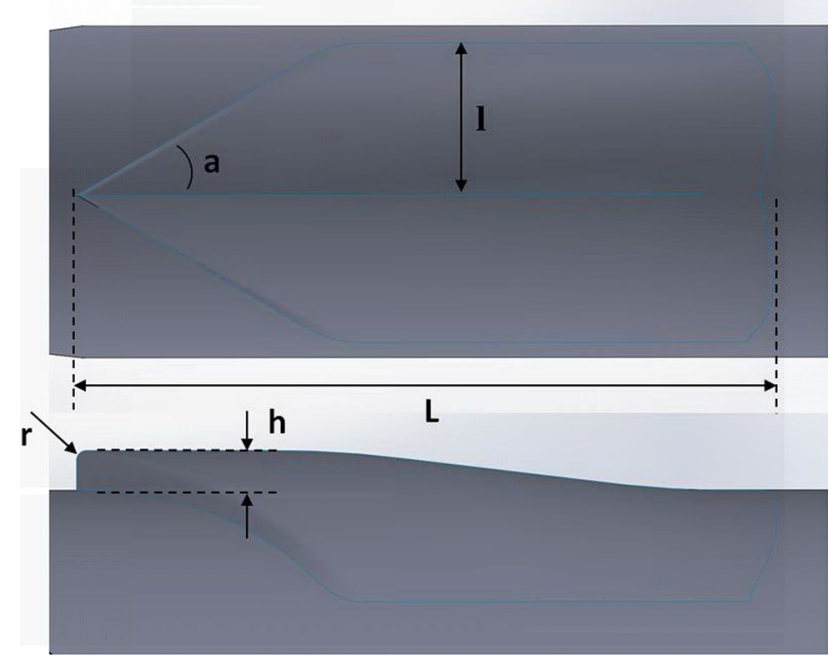

Fig. 6 ACD shape

this ACD is depicted in Fig. 6 with the most relevant geometrical parameters. Their values, made dimensionless by the total length $L$ of this ACD, are gathered in Table 1 . During the numerical optimization process, 20 shapes have been designed and analysed through RANS computations. In Table 1, the last column provides the ranges in which the key geometrical parameters have been varied in. The associated test campaign, dedicated to confirm this promising ACD shape, is detailed in the next paragraph.

\section{Experimental test campaign}

\subsection{Wind tunnel and model}

The experimental campaign has been performed in the F2 wind tunnel located at the ONERA Le Fauga-Mauzac 
Table 1 Geometrical parameters of the optimized ACD shape (dimensionless with the total length $L$ )

\begin{tabular}{llll}
\hline & & $\begin{array}{l}\text { Optimized } \\
\text { ACD }\end{array}$ & $\begin{array}{l}\text { Optimization } \\
\text { Span }\end{array}$ \\
\hline Total length & $L$ & 1 & {$[0.65,1]$} \\
Chordwise width & $l$ & 0.25 & {$[0.22,0.25]$} \\
Front height & $h$ & 0.058 & {$[0.0145,0.058]$} \\
Apex half angle & $a$ & $35^{\circ}$ & {$\left[35^{\circ}, 50^{\circ}\right]$} \\
Top front edge radius & $r$ & 0.0145 & {$[0.00145,0.0145]$} \\
\hline
\end{tabular}

centre. This wind tunnel is an atmospheric continuousflow wind tunnel with a rectangular test section $1.8 \mathrm{~m}$ high, $1.4 \mathrm{~m}$ wide and $5 \mathrm{~m}$ long. The free-stream velocity ranges from 5 to $90 \mathrm{~m} \mathrm{~s}^{-1}$ and the related turbulence level is quite low $(\mathrm{Tu} \simeq 0.07 \%$ ). The DTP-A model (see Figs. 5, 7) is a constant streamwise chord $(c=1860 \mathrm{~mm})$ and a $50^{\circ}$ swept wing ( $\operatorname{span} s=2550 \mathrm{~mm}$ ) generated from a symmetrical airfoil. It is composed of a cylindrical leading edge (radius $R=200 \mathrm{~mm}$ ) extended by a triangular shape till the trailing edge. As described in Fig. 7, a removable part of the leading edge enables the model to be outfitted with an ACD without any step junction. The leading edge of the ACD is located $600 \mathrm{~mm}$ far from the wind tunnel floor taken as the origin for the $\mathbf{Z}$ direction. This model is relevant to contamination studies because it represented in a macroscopic way a leading edge with values of $\bar{R}$ which are typically those of commercial aircraft. The floor of the wind tunnel simulates the fuselage by generating the turbulent boundary layer.

During the experiments, two configurations have been tested.

A first ACD-free configuration has enabled the validation of the experimental set-up by checking the Poll's criterion (threshold of contamination $\bar{R}=250$ ). Additionally, a configuration with ACD and roughness (tripping device) has been investigated to check whether turbulence was selfsustaining for $\bar{R}$ values higher than 250. The corresponding velocities range from 5 to $60 \mathrm{~m} \mathrm{~s}^{-1}$.

In the second configuration, the DTP-A model was equipped with the optimized shape ACD. For this configuration, a wide range of velocity values (from 10 to $90 \mathrm{~m} \mathrm{~s}^{-1}$ ) has been covered with a low velocity step so as to properly capture Görtler-Hämmerlin and crossflow instabilities (related to natural instability).

\subsection{Metrology}

Wall pressure and hot-film measurements were performed as well as infrared visualizations (see Fig. 7). Wall pressure measurements were achieved using 53 taps located on 3 lines orthogonal to the leading edge and distributed symmetrically from the attachment line:

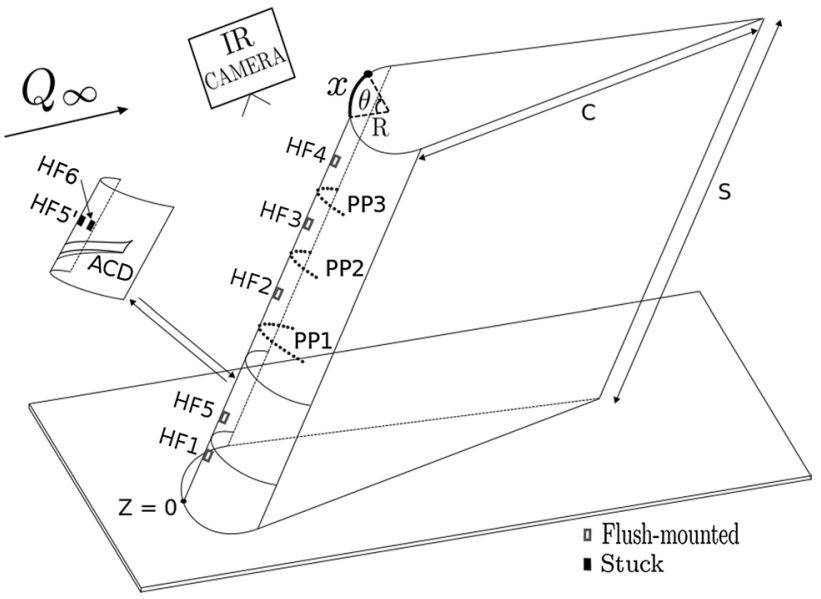

Fig. 7 Experimental set-up

Table 2 Hot-film positions for the two configurations

\begin{tabular}{llll}
\hline $\begin{array}{l}\text { ACD-free con- } \\
\text { figuration }\end{array}$ & Position $z(\mathrm{~mm})$ & $\begin{array}{l}\text { ACD configura- } \\
\text { tion }\end{array}$ & Position $z(\mathrm{~mm})$ \\
\hline HF1 & 578 & HF1 & 578 \\
HF5 & 600 & - & - \\
- & - & HF5' & 1135 \\
- & - & HF6 & $1135(x=70)$ \\
HF2 & 1510 & HF2 & 1510 \\
HF3 & 1901 & HF3 & 1901 \\
HF4 & 2395 & HF4 & 2395 \\
\hline
\end{tabular}

1. $40 \%$ span: 27 taps (PP1),

2. $60 \%$ span: 13 taps (PP2),

3. $73.5 \%$ span: 13 taps (PP3),

Hot-film measurements (HF) were also performed in order to detect the nature of the boundary layer (laminar, transitional or turbulent). Dantec 55R47 hot-films were conditioned with a home-made constant temperature anemometer. Whatever the configuration of the model, hot-films HF1 to HF5 were all flush-mounted along the attachment line in order to be as non-intrusive as possible for the flow (configuration set on the detailed model view, Fig. 7). For the configuration with ACD, the removable part of the smooth leading edge was replaced by the block with the ACD plus two additional hot-films (HF5', HF6) which were simply stuck on the model (detached block on Fig. 7). The hot-film 6 was located in a region where crossflow instabilities are alleged to occur $(x=70 \mathrm{~mm})$. The corresponding hot-film positions are given in Table 2. After AC coupling, the data acquisition system was composed of anti-aliasing low-pass filters $(10 \mathrm{kHz})$ and amplifiers (gain 20 or 100). Meas- 
urements were made at a $25 \mathrm{kHz}$ sampling rate. The test section was also equipped with an infrared camera (IRC 900) set above the wind tunnel ceiling. The heat flux between the surface and the flow is more important with a turbulent flow than a laminar one. Areas in the model where the flow is turbulent lead to a quicker temperature change. By cooling or heating punctually the flow in the wind tunnel, transition position can then be identified. An example of infrared visualization is given in Fig. 12 where lightest area represents a laminar boundary layer.

For both configurations, the only varying parameter was the wind tunnel velocity. Based on experimental measurements, the quantity $\bar{R}$ will be estimated as a function of the velocity. Next paragraph is devoted to $\bar{R}$ estimation using an intermediate quantity called effective sweep angle and denoted $\varphi_{\text {eff. }}$.

\section{Experimental results}

\subsection{Effective sweep angle and $\bar{R}$ determination}

$\varphi_{\text {eff }}$ makes reference to the sweep angle $\varphi$ that should be taken in inviscid fluid solution in order to fit as close as possible to the experimental pressure distribution on the attachment line. Due to viscous and set-up effects, stall and horse-shoe vortices, $\varphi_{\text {eff }}$ is usually different from the geometrical one. The effective sweep angle $\varphi_{\text {eff }}$ is chosen in order to recover the value of the interpolated pres-

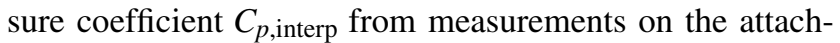
ment line $\left(\theta=0\right.$ see Fig. 8). The link between $\varphi_{\text {eff }}$ and $C_{p \text {,interp }}(\theta=0)$ is given in Eq. (11). We first start with the classical relationship giving the theoretical expression of $C_{p}$ for a swept cylinder as a function of $\varphi$ and $\theta$ :

$C_{p}(\theta)=\frac{p(\theta)-p_{\infty}}{\frac{1}{2} \rho_{\infty} Q_{\infty}^{2}}=\cos ^{2}(\varphi)\left(1-4 \sin ^{2}(\theta)\right)$

where $p_{\infty}$ and $\rho_{\infty}$ are, respectively, the inflow pressure and density. Fitted experimental pressure coefficient around $\theta \approx 0$ is then used to evaluate $\varphi_{\text {eff }}$ :

$C_{p, \text { interp }}(\theta=0)=\cos ^{2}\left(\varphi_{\text {eff }}\right)$

$\varphi_{\mathrm{eff}}=\cos ^{-1}\left(\sqrt{C_{p, \text { interp }}(\theta=0)}\right)$

The evolution of the effective sweep angle in spanwise direction is illustrated in Fig. 9. Measurements (symbols) have been plotted at locations corresponding to the wall pressure taps (PP1, PP2, PP3) for three values of the inflow velocity $Q_{\infty}$. The geometrical sweep angle $\left(\varphi_{\text {geom }}=50^{\circ}\right)$ is represented by the horizontal dashed line. The difference between effective and geometrical angles varies from $6^{\circ}$ to $8.5^{\circ}$ and

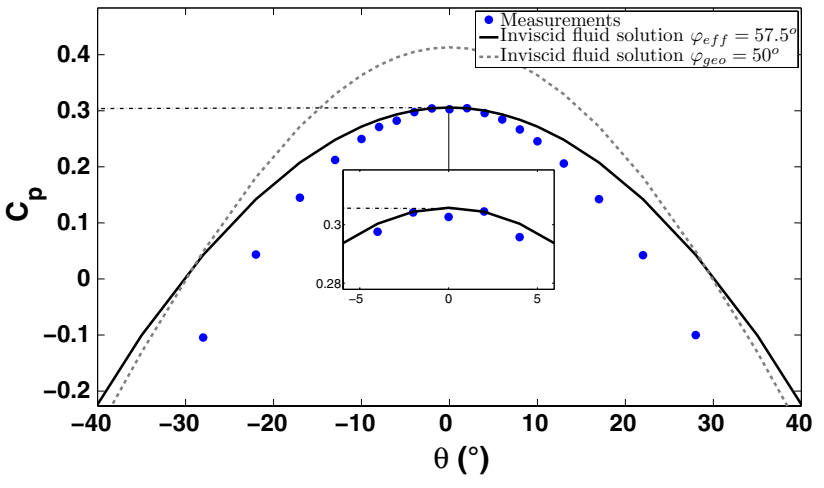

Fig. 8 Experimental and inviscid fluid solution for wall pressure coefficient (PP1) at $Q_{\infty}=75 \mathrm{~m} \mathrm{~s}^{-1}, C_{p \text {,interp }}(\theta=0)=0.31$

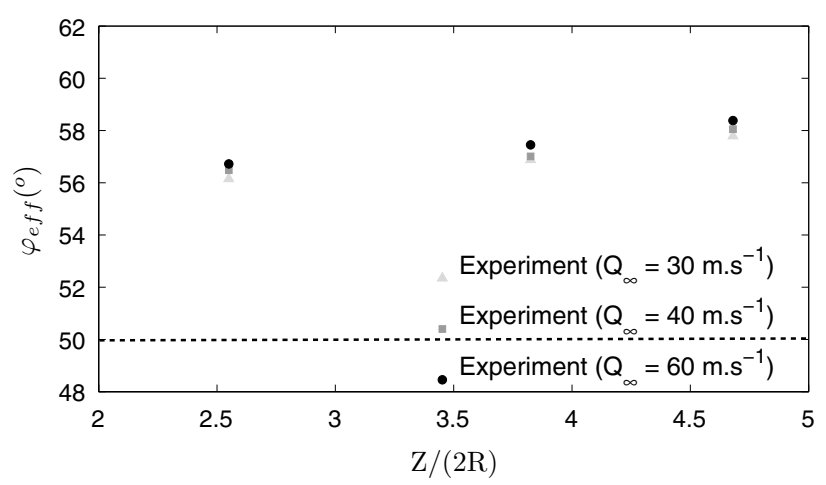

Fig. $9 \varphi_{\text {eff }}$ along span for different free-stream velocities, $\varphi_{\text {geo }}=50^{\circ}$

increases with the inflow velocity. This demonstrates that wall effects are significant. Figure 8 depicts a typical distribution of pressure coefficient around the leading edge. Measurements (circles) were made at $Z / 2 R=2.55$ (PP1) for an inflow velocity $Q_{\infty}=75 \mathrm{~m} \mathrm{~s}^{-1}$. Two additional curves have been plotted. The dashed curve corresponds to $C_{p}$ evolution provided by Eq. (10) using the geometrical sweep angle $\varphi_{\text {geom }}$, whereas the full line stands for $C_{p}$ values obtained with the effective sweep angle $\varphi_{\text {eff }}=57.5^{\circ}$. In the vicinity of the attachment line, measurements match with the $\varphi_{\text {eff }}$ curve, but further downstream, the geometrical angle gives best results. Rigorous characterization of effective sweep angle is of prime importance to provide accurate values of $\bar{R}$. Indeed, under the assumption of a cylindrical leading edge $\left(U_{e}=2 U_{\infty} x / R\right)$, it can be demonstrated that $\bar{R}$ (defined in Eq. 5) becomes a function of $\varphi_{\text {eff }}$ which has been estimated previously:

$\bar{R}=\sqrt{\frac{Q_{\infty} R \sin \left(\varphi_{\text {eff }}\right) \tan \left(\varphi_{\text {eff }}\right)}{2 v}}$

where $R$ is the radius of the cylindrical leading edge. Leading edge of the experimental model being cylindrical, this approach has been applied to determine corresponding $\bar{R}$ 


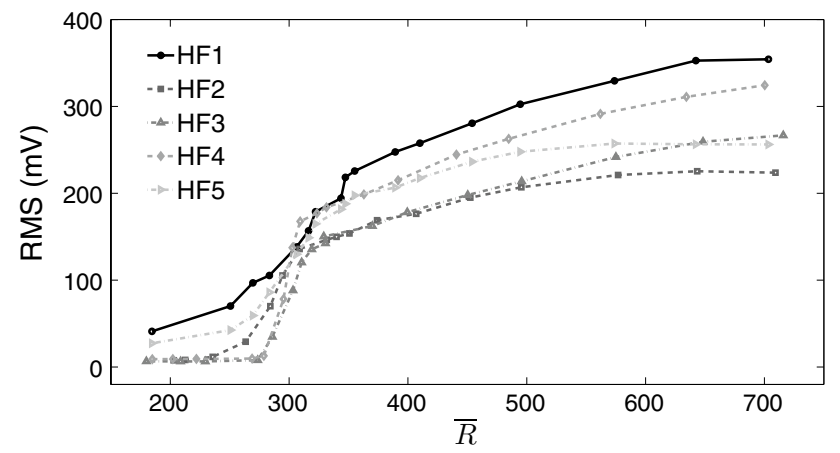

Fig. 10 Experimental hot-film RMS values as a function of $\bar{R}$ for the reference configuration (without ACD)

values for each pressure measurement section. According to experimental results, $\varphi_{\mathrm{eff}}$ is likely to increase slightly along span (see Fig. 9) so that $\bar{R}$ increases correspondingly.

It can be noticed that this approach for $\bar{R}$ estimation can be extended to all airfoil considering that any leading edge can be approached by its osculating circle.

\subsection{Validation of Poll's criterion}

Contamination occurrence and transition locations are achieved using hot-film measurements. Contamination and transition from a laminar to a turbulent boundary layer lead to a strong increase in the recorded RMS values of the hotfilm signal. Hot-films being spread all along the attachment line, transition location can thus be estimated.

As mentioned previously, an ACD-free configuration has been used in order to check the compliance of Poll's criterion. Figure 10 shows high RMS values for HF1 that can be explained by the fact that this hot-film is down enough to be permanently under the influence of the turbulent boundary layer which develops on the wind tunnel floor. This boundary layer has been measured at the entry of the test section during previous campaigns and has a maximum thickness of $100 \mathrm{~mm}$ for an upstream velocity of $12 \mathrm{~m} \mathrm{~s}^{-1}$. The HF1 signal will be used as a reference for RMS values related to turbulent boundary-layer flow. Elsewhere, for hot-films 2 to 5 located higher on the attachment line, Fig. 10 shows a strong increase in RMS values around $\bar{R}=250-280$ which means that contamination has just occurred. This clearly shows a good agreement of Poll's criterion with the current experimental set-up.

Another way to demonstrate that our set-up was compliant with the Poll's criterion was to destabilize the new laminar boundary layer on the ACD surface using a small roughness element (Zigzag tape $700 \mu \mathrm{m}$ thick). An infrared visualization has been performed to highlight the nature of the flow downstream the roughness at values of $\bar{R}$ close to 250.
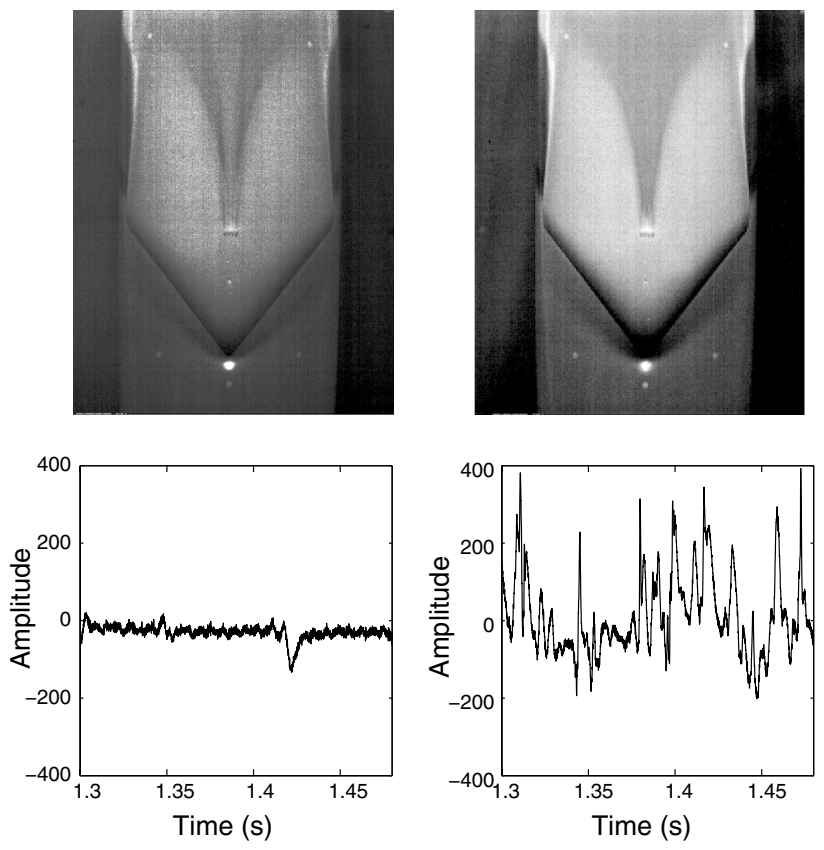

Fig. 11 Infrared visualizations of ACD with roughness at $\bar{R}=237$ (left) and $\bar{R}=263$ (right) with below the corresponding HF2 RMS signals

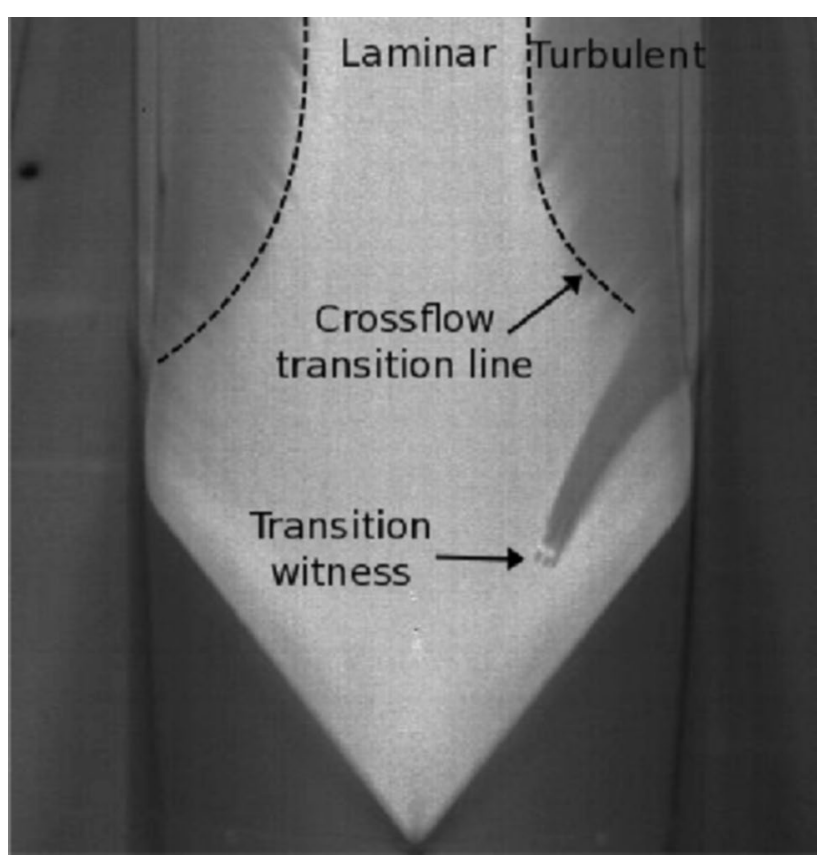

Fig. 12 Infrared visualization of the ACD at $Q_{\infty}=60 \mathrm{~m} \mathrm{~s}^{-1}$, $\bar{R}=650$ with tripping element (transition witness)

For values of $\bar{R}$ slightly lower than $250(\bar{R}=237)$, a laminar region can be observed (represented by a lighter central area) downstream the roughness and low RMS values for the hot-film located in this wake (Fig. 11 left). 
For values of $\bar{R}$ slightly higher than $250(\bar{R}=263)$, a uniform colour density area downstream the roughness is observed and high RMS values indicate a whole turbulent area (see Fig. 11 right). Isolated roughness criteria ${ }^{2}$ have been applied and tend to show that the roughness is not high enough to be responsible for the transition to turbulence observed.

Hot-film measurements on this smooth configuration and the observations done with the small roughness element confirm Poll's criterion about the critical value of $\bar{R}$ and are likely to show the self-sustainability of turbulence at values higher than this threshold.

\subsection{Validation of ACD efficiency}

The second configuration aims at evaluating the efficiency of the ACD to prevent leading-edge contamination. Low RMS values are recorded for the hot-films distributed along the attachment line downstream the ACD (see Fig. 13, HF2 to HF4) around $\bar{R} \simeq 250$, whereas RMS values were already high at this same $\bar{R}$ in smooth condition (ACD-free configuration).

Furthermore, RMS values remain low up to $\bar{R} \simeq 580$ for hot-films HF3 and HF4. Along the attachment line, without contamination, the linear stability theory developed in Sect. 2.3 established that natural transition could occur as soon as $\bar{R}=582$ (Görtler-Hämmerlin approach). This makes possible to conclude that the ACD fully plays its role allowing the downstream development of a new laminar boundary layer until the inherent natural destabilization which represents the maximal value that can be reached without using any other stabilizing devices (wall suction for example).

This ACD even confirms its ability to prevent leadingedge contamination at $\bar{R}$ values higher than 580. Indeed, according to the infrared visualization presented in Fig. 12 with a transition witness (Zigzag tape $400 \mu \mathrm{m}$ thick), the boundary layer downstream the ACD is still laminar at $\bar{R}=650$ showing that no turbulent structure has come across the ACD. This observation is confirmed on Fig. 13 for the closest hot-film HF5' that still exhibits low RMS values at this $\bar{R}$. It can be noticed that HF5' and HF6 record low RMS values related to a laminar boundary layer at values of $\bar{R}$ higher than the Görtler-Hämmerlin critical $\bar{R}$. These two hot-films being close to the ACD, the spatial amplification has to be enhanced (higher velocities, i.e. higher values of $\bar{R}$ ) so that instabilities reach a critical threshold and trigger the transition. HF6 is transitional at lower values than HF5' due to a crossflow transition as

$\overline{2 \text { van Driest }}$ and Blumer (1962) and von Doenhoff and Braslow (1961) criteria.

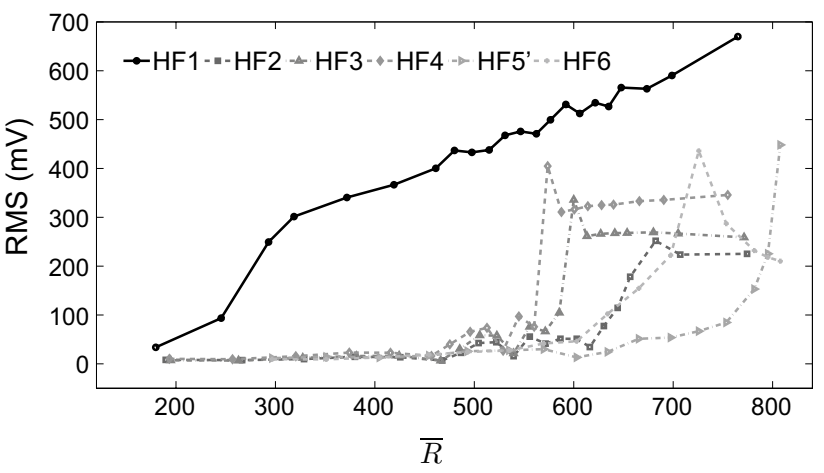

Fig. 13 Experimental hot-film RMS values along $\bar{R}$ for the ACD configuration

explained in the following section. Relatively to the best existing ACDs and Gaster bumps that were efficient up to $\bar{R} \simeq 450$, this ACD shows a significant improvement in the anti-contamination ability.

A characterization of natural leading edge instability observed in this ACD configuration is proposed in the next section and will make possible to confirm that transition process observed for HF2 to HF4 at $\bar{R} \simeq 580$ is due to a natural transition.

\subsection{Natural transition process downstream the ACD}

\subsubsection{Görtler-Hämmerlin transition process}

Time-resolved hot-film measurements performed just before transition onset enable to perform some spectral analyses. Frequencies of amplified waves have been estimated by extracting the amplitude spectrum of the signal using a Fast Fourier Transform (FFT). In order to define the link between these amplified frequencies and GörtlerHämmerlin instabilities, spectra have been compared to the stability approach described in Sect. 2.3.

Referring to the form of a perturbation introduced in Eq. (9b), the amplitude A (norm) of a perturbation can be written as:

$A=\left\|q^{\prime}\right\|=\frac{1}{\sqrt{2}}\|q(x, y)\| \exp \left(-\beta_{i} Z\right)$

where $q^{\prime}=u^{\prime}, v^{\prime}, w^{\prime}, \pi^{\prime}$ and $q=x u, v, w, \pi$

Using exponential properties, this leads to:

$\frac{\mathrm{d} A}{A}=-\beta_{i} Z$

Assuming a reference amplitude $A_{0}$ as known, amplification can be written as:

$\frac{A}{A_{0}}=\exp \left(\int-\beta_{i} \mathrm{~d} Z\right)$ 


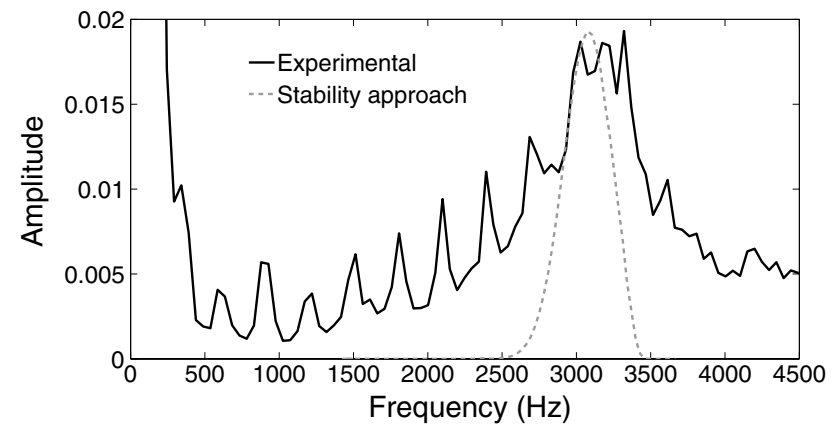

Fig. 14 Experimental versus stability approach for $\mathrm{HF}^{\prime}$ at $Q_{\infty}=52.5 \mathrm{~m} \mathrm{~s}^{-1}, \bar{R}=621$

Under the assumption of an infinite wing $(\partial / \partial Z=0)$, the amplification can be directly written as:

$\frac{A}{A_{0}}=\exp \left(-\beta_{i} Z-Z_{0}\right)$

where $Z_{0}$ is a reference position and $A_{0}$ the amplitude of the disturbances at this location. For sake of simplicity, it will be considered in the following that $Z_{0}=0$ and corresponds to the nose of the ACD. For any experimental hot-film spectrum where $\bar{R}$ is known, $\omega=2 \pi f$ is made varying to meet the interval of experimental frequency band $[0,12,500 \mathrm{~Hz}]$. For each couple $(\bar{R}, \omega)$, the stability approach gives the imaginary part $\beta_{i}$ of Görtler-Hämmerlin mode. The position $Z$ of the hot-film being known, $A / A_{0}=\exp \left(-\beta_{i} Z\right)$ can be calculated for each frequency. It remains that experimental spectrum is expressed in terms of amplitude $(A)$, whereas the numerical one is expressed in terms of amplification $\left(A / A_{0}\right)$. However, hot-films were not calibrated during the experimental test campaign and had just been used to qualitatively analyse the state of the boundary layer. This means that HF signal cannot be employed to quantify amplitude of instabilities in the laminar region of the boundary layer. As a consequence, in order to compare stability theory to measurements, numerical results have been rescaled to fit with the maximum value of the voltage fluctuation.

Agreement in amplified frequencies between experimental and stability approach is good (see Figs. 14, 15). This tends to confirm that the destabilization of the flow is due to natural transition process involving Görtler-Hämmerlin instabilities.

In laminar-turbulent transition studies, a common expectation is the ability to determine or predict transition location. To this end, Smith and Gamberoni (1956) as well as van Ingen (1956) developed a semi-empirical method, the so-called $e^{N}$ method, that links the transition triggering to a critical value of the $N$ factor. This approach makes the assumption that natural transition occurs quickly after the nonlinear phase. The $N$ factor corresponds, for a fixed $\bar{R}$, to

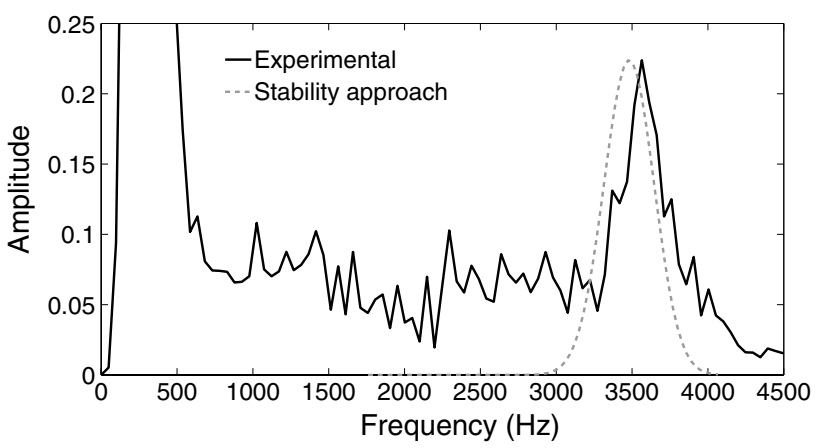

Fig. 15 Experimental versus stability approach for HF2 at $Q_{\infty}=55 \mathrm{~m} \mathrm{~s}^{-1}, \bar{R}=636$

the most amplified wave $A / A_{0}$ expressed in terms of natural logarithm:

$N=\max \left[\ln \left(\frac{A}{A_{0}}\right)\right]=\int-\beta_{i, \max } \mathrm{d} Z$

$=-\beta_{i, \max } Z$

According to this expression, if the value of $N$ at transition is known, it makes possible to estimate the transition position along span $(Z)$. Depending on the inflow velocity, a hot-film signal may be laminar (for a given value of $\bar{R}_{\text {lam }}$ ) or turbulent (for a higher value of $\bar{R}_{\text {turb }}$ ), $\overline{R_{\mathrm{cr}}}$ being between these two values. Associated $-\left(\beta_{i}\right)_{\max }$ is estimated using the stability approach. It is then possible to give a range for the critical $N$ factor associated with Görtler-Hämmerlin instability along the attachment line and corresponding to transition onset.

The $N$ factor range is estimated using HF2 and HF5. Experimental results (see Table 3 ) give a possible interval for the $N_{T}$ factor of $[7.2,10]$ for hot-film 2 and $[7.1,7.8]$ for hot-film 5 .

\subsubsection{Crossflow transition process}

In the experiment, hot-film 6 has been set up slightly shifted from the attachment line, in region where crossflow instabilities are alleged to occur $(x=70 \mathrm{~mm})$. Spectra have been extracted from the temporal signal of HF6 in order to study the frequency of instabilities developing inside the boundary layer at this chordwise location. The aim is to compare these frequencies to the one provided by the linear stability theory. The chordwise evolution of $\mathrm{N}$ factors for distinct frequencies and an upstream velocity $Q_{\infty}=50 \mathrm{~m} \mathrm{~s}^{-1}$ is represented in Fig. 16. It demonstrates that unsteady crossflow modes are more amplified than their stationary counterpart. At HF6 location (represented by the vertical dashed line), the $N$ factor is $N=3.6$ for the stationary CF mode, while it reaches $N=7.2$ for $f=2$ 
Table 3 Görtler-Hämmerlin critical $N$ factor values around transition onset

\begin{tabular}{lll}
\hline & Hot-film 2(Z=0.910) & Hot-film 5 $(Z=0.535)$ \\
\hline $\bar{R}_{\text {lam }}-\bar{R}_{\text {turb }}$ & $682-707$ & $721-732$ \\
$-\beta_{i, \text { max }}$ & $7.9,11.0$ & $13.3,14.6$ \\
$N$ & {$[7.2,10]$} & {$[7.1,7.8]$} \\
\hline
\end{tabular}

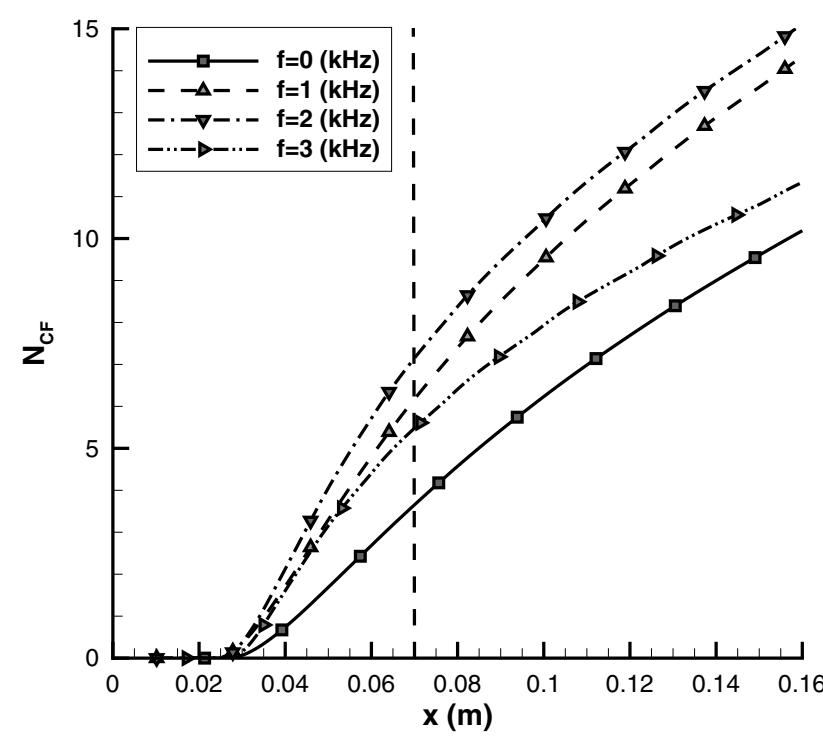

Fig. 16 Spatial evolution of crossflow instability $N$ factors for various frequencies: $f=0,1,2,3(\mathrm{KHz})$ for $Q_{\infty}=50 \mathrm{~m} \mathrm{~s}^{-1}$. The vertical dashed line corresponds to HF6 location

$\mathrm{kHz}$. From a receptivity point of view, stationary CF vortices are initiated by wall-distributed roughness elements. Travelling modes are generated by a combined effect of external turbulence fluctuations and wall roughness elements. Bippes (1999) has shown that stationary and travelling modes should be in the same order of magnitude for $T u \simeq 0.1 \%$. In the current experiment, the turbulence level is close to this value so that a possible competition between steady/unsteady modes may exist. This is shown by the experimental spectrum for $Q_{\infty}=50 \mathrm{~m} \mathrm{~s}^{-1}$ (full line in Fig. 17) which demonstrates that unsteady instabilities propagate inside the boundary layer. It has been compared to the numerical spectrum (dashed line in Fig. 17) obtained from linear stability theory. Numerical levels have been scaled in order to fit with the measurements. Wave amplification given by stability analysis is in good agreement with experimental one, despite a wider numerical range of amplified frequencies.

Crossflow vortices are amplified at low frequency and correspond to what is commonly admitted for this type of flow destabilization. Transition onset results from a competition between steady/unsteady modes depending on the

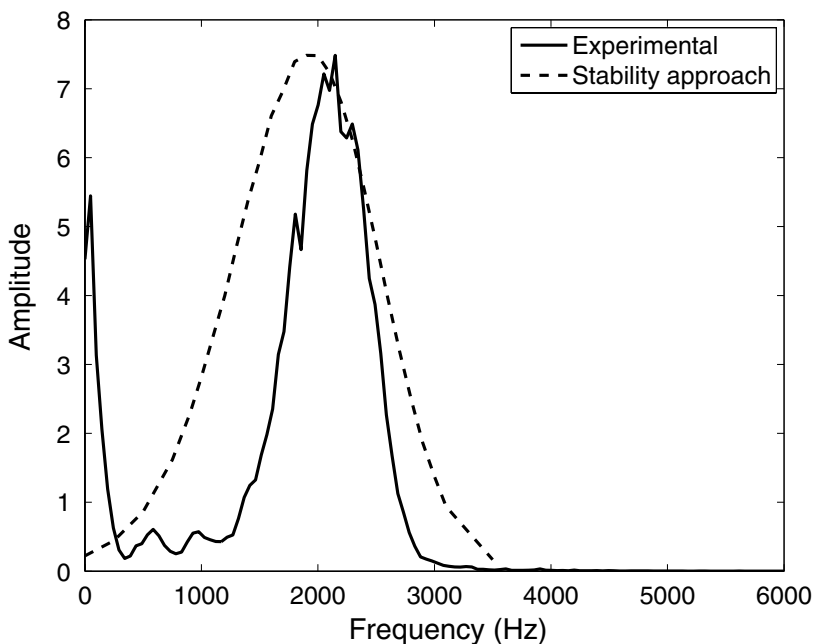

Fig. 17 Experimental versus stability approach spectrum for HF6 and for $Q_{\infty}=50 \mathrm{~m} \mathrm{~s}^{-1}$

receptivity and amplification phases. Crossflow transition line is visible in Fig. 12

\section{Conclusion}

This paper deals with attachment line transition processes that may occur on real swept wings of commercial aircraft and specially the contamination issue. In this framework, ONERA developed an entire procedure to design a highefficiency anti-contamination devices (ACDs) which can be fitted on various realistic applications. This goes from the parametrized conception of the shape to the validation of the anti-contamination efficiency using both numerical and experimental approaches. The optimized design of a chevron-shaped ACD resulting from this procedure has been tested in a wind tunnel and its efficiency is assessed. This ACD has shown its ability to prevent leading-edge contamination up to values of $\bar{R}$ close to the natural transition process, i.e. the maximal value that can be expected without additional stabilizing process. The ability of this ACD to let the development of a laminar boundary layer downstream has made possible to characterize natural instabilities along the attachment line, preferential amplified frequencies and $\mathrm{N}$ factor of transition. This optimized shape ACD is an important step to deal with laminar maintenance of boundary layer along the attachment line of commercial aircraft evolving at high $\bar{R}$ values.

It corresponds to the first necessary condition to the maintenance of a laminar boundary layer as far as possible around aircraft airfoil where further downstream other mechanisms can lead to turbulence, especially crossflow vortices and Tollmien-Schlichting destabilizing waves. $\bar{R}$ 
values of common commercial aircraft being usually higher than the natural stability one, a promising way to maintain a laminar flow along the leading edge at higher $\bar{R}$ would be to couple this ACD with a suction system along the attachment line that could delay the natural transition. Contamination process is then prevented by the ACD, and natural transition process can be moved forward thanks to suction delaying the transition at higher $\bar{R}$.

Acknowledgments Part of this work has been undertaken within the Joint Technology Initiative «JTI Clean Sky» and specifically in the framework of the Smart Fixed Wing Aircraft Integrated Technology Demonstrator (SFWA-ITD Contract No. CSJU-GAMSFWA-2008-001) financed by the 7th Framework Program of European Commission. The authors would like to thank the partners of the project for their collaboration and fruitful discussions. The authors are grateful to ONERA/GMT F2 wind tunnel team J.M. Deluc, T. Joret and P. Loiret for their technical support during the experimental campaign in F2 wind tunnel. The authors would also like to warmly thank F. Ternoy and G. Outtier who manufactured the ACD. Special thanks are given to D. Arnal and A. Séraudie who initiated this study and performed the first wind tunnel campaign in 2010 (they well deserved their retirement).

\section{References}

Arnal D (1996) Control of laminar turbulent transition for skin friction drag reduction. In: Control of flow instabilities and unsteady flows. In: Meier GEA, Schnerr GH (eds) CISM Courses and lectures, vol 369. Springer-Verlag, New York, pp 119-153

Arnal D, Habiballah M, Coustols E (1984) Laminar instability theory and transition criteria in two- and three-dimensional flows. La Recherche Aérospatiale (English Edition), 2nd edn. pp 45-63

Arnal D, Reneaux J, Preist J (1996) Control of attachment line contamination. In: 2nd European forum on laminar flow technology, Bordeaux

Bippes H (1999) Basic experiments on transition in three-dimensional boundary layers dominated by crossflow instability. Prog Aerosp Sci 35(4):363-412. doi:10.1016/S0376-0421(99)00002-0
Gasparian G (1998) Experimental study and transition mechanisms modeling on swept wing, with application to laminar flow control. Ph.D. thesis, ENSAE

Gaster M (1967) On the flow along swept leading edges. Aeronaut Quat 18(2):165-184

Görtler H (1955) Dreidimensionale instabilität der ebenen staupunktströmung gegenüber wirbelartigen störungen. Fifty years of boundary layer research. Vieweg und Sohn, Braunschweig, p 304

Hiemenz K (1911) Die grenzschicht an einem in den gleichförmigen flüssigkeitsstrom eingetauchten geraden kreiszylinder. Dingl. Polytech., Göttingen, Phil. Diss

Krier JV, Sucipto T (2009) Highly optimizable laminar flow control devices. In: 19th AIMETA Congress, Ancona, Italy

Krier JV, Sucipto T, Archambaud JP, Godard JL, Donelli R, Arnal D (2009) Passive and active device for laminar flow control of swept wing. In: Proceedings KATNET II Conference on Key Aerodynamic Technologies. Bremen, Germany

Morkovin M, Reshotko E, Herbert T (1994) Transition in open flow systems - a reassessment. Bull of the Am Phys Soc 39(9):1882-1994

Pfenninger W (1965) Flow phenomena at the leading edge of swept wings. Recent Developments in Boundary Layer Research. AGARDograph 97, Part IV

Poll D (1978) Some aspects of the flow near a swept attachment line with particular reference to boundary layer transition. Tech. rep., College of Aeronautics

Poll D (1985) Boundary layer transition on the windward face of space shuttle during re-entry. AIAA paper 85-0899

Smith A, Gamberoni N (1956) Transition, pressure gradient and stability theory. Technical report ES-26388, Douglas Aircraft Company

von Doenhoff A, Braslow A (1961) Effect of distributed surface roughness on laminar flow. In: Lachmann GV (ed) Boundary layer control, vol 2. Pergamon Press, New-York, pp 657-681

van Driest E, Blumer C (1962) Boundary layer transition at supersonic speeds. Three-dimensional roughness effects (spheres). J Aerosp Sci 29:909-916

van Ingen J (1956) A suggested semi-empirical method for calculation of the boundary layer transition region. University of Technology, Department of Aerodynamics UTH-74, Delft 